\title{
Some Characterizations of Weighted Holomorphic Function Classes by Univalent Function Classes
}

\author{
A. El-Sayed Ahmed $\mathbb{D}^{1}$ and S. Omran ${ }^{2,3}$ \\ ${ }^{1}$ Mathematics Department, Faculty of Science, Taif University, P.O. Box 11099 Taif 21944, Saudi Arabia \\ ${ }^{2}$ Mathematics Department, Faculty of Science, South Valley University, P.O. Box 83521 Qena, Egypt \\ ${ }^{3}$ The Academy of Scientific Research and Technology (ASRT), Cairo, Egypt
}

Correspondence should be addressed to A. El-Sayed Ahmed; ahsayed80@hotmail.com

Received 10 December 2020; Accepted 22 February 2021; Published 11 March 2021

Academic Editor: Shuangping Tao

Copyright (c) 2021 A. El-Sayed Ahmed and S. Omran. This is an open access article distributed under the Creative Commons Attribution License, which permits unrestricted use, distribution, and reproduction in any medium, provided the original work is properly cited.

Some characterizations of $Q_{K, \omega}(p, q)$ - type classes of holomorphic functions by Schwarzian derivatives with known conformal-type mappings are introduced in the present manuscript. Moreover, the action of the pre-Schwarzian derivatives on $Q_{K, \omega}(p, q)-$ type classes, typically the univalent ones by using concerned Carleson-type measures, is investigated. In addition, we reveal important characterizations of some concerned weighted analytic-type spaces with the known Schwarzian derivatives evolving certain $Q$ - type of concerned function class for a high utility toward practical and feasible application of concerned domains.

\section{Introduction}

Some concepts and terminologies related to weighted holomorphic function spaces are briefly recalled in this concerned section.

Suppose that $\mathbb{D}=\{w \in \mathbb{C}: 0<|w|<1\}$ defines the open unit disc in $\mathbb{C}$. The class which involves all holomorphic functions in $\mathbb{D}$ is symbolized by $\mathscr{H}(\mathbb{D})$. Assume that $d \sigma_{w}=$ $d x d y$ defines the usual normalized area measure on $\mathbb{D}$. Suppose that the known Green's function on $\mathbb{D}$ is given by $g(w, a)=\log \left(1 /\left|\varphi_{a}(w)\right|\right)$, with $\varphi_{a}(w)=w-a / 1-\bar{a} w$, for $w, a \in \mathbb{D}$. The specific class of all known univalent functions in $\mathbb{D}$ will be symbolized by $\mathcal{U}$ (see [1-3] and others). If $\in \mathcal{U}$, $Q=g(\mathbb{D})$, and $\partial Q$ stands for the Jordan curve, so $g: \mathbb{D}$ $\longrightarrow Q$ will be called conformal map [2]. Throughout this manuscript, the function $\omega:(0,1] \longrightarrow[0, \infty)$ with $\omega \neq 0$. In addition, the concerned function $K:[0, \infty) \longrightarrow[0, \infty)$ stands for a right-continuous and nondecreasing specific function.

Next, we report the recent advancements of the concepts of specific weighted classes of holomorphic function spaces. The choice of the appropriate functions give the specific essential properties of the underlying weighted classes of functions can have an important impact for the study.

Suppose that

$$
\begin{gathered}
T(\alpha, \omega)=\frac{(1-|w|)^{\alpha}}{\omega(1-|w|)} \\
T(K(g(w, a)), \omega)=\frac{K(g(w, a))}{\omega(1-|w|)} .
\end{gathered}
$$

Definition 1 [4-6]. Let $0<\alpha<\infty$. Then, the function $g \in \mathscr{H}$ $(\mathbb{D})$ is including in $\alpha$-weighted Bloch-type space $\mathscr{B}_{\omega}^{\alpha}$ when

$$
\|g\|_{\mathscr{B}_{\omega}^{\alpha}}=\sup _{w \in \mathbb{D}} T(\alpha, \omega)\left|g^{\prime}(w)\right|<\infty
$$

Definition 2 [4-6]. Let $0<\alpha<\infty$. The function $g \in \mathscr{H}(\mathbb{D})$ is included in the little specific weighted Bloch-type space $\mathscr{B}_{\omega, 0}^{\alpha}$ when 


$$
\|g\|_{\mathscr{B}_{\omega, 0}^{\alpha}}=\lim _{|w| \rightarrow 1^{-}} T(\alpha, \omega)\left|g^{\prime}(w)\right|=0 .
$$

When $p \in(0, \infty), q \in(-2, \infty)$, let $Q_{K, \omega}(p, q)$ be the concerned analytic space that consists of all specific functions $g \in \mathscr{H}(\mathbb{D})([5,6])$, for which

$\|g\|_{\mathscr{Q}_{K, \omega}(p, q)}^{p}:=\sup _{a \in \mathbb{D}} \int_{\mathbb{D}}\left|g^{\prime}(w)\right|^{p}\left(1-|w|^{2}\right)^{q} T(K(g(w, a)), \omega) d \sigma_{w}<\infty$.

Further, on the boundary of $\mathbb{D}$, we can say that $g \in Q_{K, \omega}$ $(p, q)$ belongs to the concerned class $Q_{K, \omega, 0}(p, q)$ when

$$
\lim _{|a| \rightarrow 1} \int_{\mathbb{D}}\left|g^{\prime}(w)\right|^{p}\left(1-|w|^{2}\right)^{q} T(K(g(w, a)), \omega) d \sigma_{w}=0
$$

Remark 3. For more research on $Q_{K, \omega}(p, q)$, we refer to [7-11] and others. When $\omega(t) \equiv 1$, we obtain the classes $Q_{K}(p, q)$ and $Q_{K, 0}(p, q)$ (see $[12,13]$ and others). Also, there are some recent research in Clifford setting (see [14-17] and others).

In this manuscript, we dealt with discussing Schwarzian derivative class on $Q_{K, \omega}(p, q)$ classes impulsive problems of conformal mappings which is due to the derivative terms in the definitions of both weighted Bloch and $Q_{K, \omega}(p, q)$ classes of analytic-type functions. The characterizations involved Carleson measures of $K, \omega$-type with a combination of the behavior of conformal mappings between the weighted Q-type functions. For a holomorphic function in the unit disc, the Schwarzian derivative is introduced early in 1873. This derivative was given to generalize and extend the Schwarz-Christoffel derivative type to study some properties of mappings that preserve certain angles. Recently, some authors have used Schwarzian derivative to characterize some results for holomorphic classes of function spaces (see $[1,18-23]$ and others).

Hereafter, the holomorphic function $g(w)$ stands for a conformal map, and we shall set $f(w)=: \ln \left(g^{\prime}\right)(w)$. The symbol $N_{g}(w)$ denotes the pre-Schwarzian differentiability of $g(w)$, that is

$$
N_{g}(w)=: \frac{d f(w)}{d w}=\frac{g^{\prime \prime}(w)}{g^{\prime}(w)}
$$

The Schwarzian differentiability for the function $g$ is defined by

$S_{g}(w)=N_{g}^{\prime}(w)-\frac{1}{2}\left(N_{g}(w)\right)^{2}=\left(\frac{g^{\prime \prime}(w)}{g^{\prime}(w)}\right)^{\prime}-\frac{1}{2}\left(\frac{g^{\prime \prime}(w)}{g^{\prime}(w)}\right)^{2}$.

Some essential basic properties of $N_{g}(w)$ and $S_{g}(w)$ are stated in [2].
(1) When the function $g(w)$ is univalent on $\mathbb{D}$, thus

$$
\left(1-|w|^{2}\right)\left|N_{g}(w)\right| \leq 6<e^{2} \text { and }\left(1-|w|^{2}\right)^{2}\left|S_{g}(w)\right| \leq 6
$$

(2) If $\left(1-|w|^{2}\right)\left|N_{g}(w)\right| \leq 1$, or $\left(1-|w|^{2}\right)^{2}\left|S_{g}(w)\right| \leq 2$, then $g$ is univalent function on $\mathbb{D}$

(3) For all concerned functions $f \in H(\mathbb{D})$, we have that $f \in \mathscr{B} \Longleftrightarrow$, we can find $w \in \mathbb{C}$ and a concerned univalent function $g$, with $f=w \ln g^{\prime}$

(4) The concerned Schwarzian-type derivative is a Möbius invariant using the equality $S_{\varphi_{a} \circ g}=S_{g}$, and it is also satisfying $\left(1-|w|^{2}\right)^{2}\left|S_{g_{\circ} \varphi_{a}}(w)\right|=$ $\left(1-\left|\varphi_{a}(w)\right|^{2}\right)^{2}\left|S_{g}\left(\varphi_{a}(w)\right)\right|$, for every Möbius transformation $\left.\varphi_{a}(w)\right), a \in \mathbb{D}$.

Let the symbol $I \subset \partial \mathbb{D}$ define a subarc of the boundary of $\mathbb{D}$. Assume that

$$
S_{1}(I)=\{r \zeta \in \mathbb{D}: 1-|I|<r<1, \zeta \in I\}
$$

when $|I| \geq 1$, thus by letting $S_{1}(I)=\mathbb{D}$. The specific positive measure $\mu$ is said to be an actually bounded $K, \omega$ -Carleson-type measure on the disc $\mathbb{D}$ when

$$
\sup _{I \subset \partial \mathbb{D}} \int_{S_{1}(I)} \frac{K(1-|z| /|I|)}{\omega(1-|z|)} d \mu(z)<\infty
$$

Moreover, when

$$
\lim _{|I| \rightarrow 0} \int_{S(I)} \frac{K(1-|z| /|I|)}{\omega(1-|z|)} d \mu(z)=0
$$

thus $\mu$ is an actually compact Carleson-type measure of $K, \omega$-type.

Remark 4. It is very clearly to see that, if $K(t)=t^{p}$, and $\omega(1-|z|) \equiv 1$, then $\mu$ is an actually bounded p-Carlesontype measure on $\mathbb{D} \Longleftrightarrow\left(1-|w|^{2}\right) d \mu$ and an actually bounded p-Carleson-type measure on $\mathbb{D}$ (see [24]).

The next generic concerned result can be proved as the corresponding result in [25].

Lemma 5. A concerned positive measure $\mu$ on $\mathbb{D}$ is a concerned Carleson measure of $K, \omega$-type $\Longleftrightarrow$

$$
\sup _{a \in \mathbb{D}} \int_{\mathbb{D}} \frac{K\left(1-\left|\varphi_{a}(z)\right|^{2}\right)}{\omega(1-|z|)} d \mu(z)<\infty
$$

Some general concepts are defined in the following. 
Let $n=1,2, \cdots$, then the concerned specific Carleson boxes of dyadic-type are then defined by:

$B_{n, m}=\left\{z=r e^{i \theta} \in \mathbb{D}: 1-\frac{1}{2^{n}} \leq|w|<1, \frac{m}{2^{n+1}} \leq \frac{\theta}{\pi}<\frac{m+1}{2^{n+1}}\right\}, 0 \leq m \leq 2^{n+1}$,

of side-length $\ell\left(B_{n, m}\right)=1 / 2^{n}$ and their inner half

$$
T\left(B_{n, m}\right)=B_{n, m} \cap\left\{w \in B_{n, m}: 1-\frac{1}{2^{n}} \leq|w|<\frac{1}{2} \ell\left(B_{n, m}\right)\right\} .
$$

For the concerned univalent function $g$, assume that $\delta$ and $\varepsilon$ are small enough. If $B$ is a Carleson-type box of dyadic-type, then it is called that $B$ is bad when

$$
\sup _{z \in T(B)}\left(1-|w|^{2}\right)\left|N_{g}(w)\right| \geq \varepsilon \text { also } \sup _{w \in T(B)}\left(1-|w|^{2}\right)^{2}\left|S_{g}(w)\right| \leq \delta .
$$

Also, $B$ is said to be a specific maximal-type bad square when the next specific bigger concerned dyadic square $\tilde{B}$ including $B$ either has the specific equality $\ell(\tilde{B})=1 / 2$ or has the concerned inequality $\sup _{w \in T(B)}\left(1-|w|^{2}\right)^{2}\left|S_{g}(w)\right|>\delta$.

Lemma 6 [22]. Let $g$ be a concerned univalent function on the disc $\mathbb{D}$, and assume that there exists $g_{0} \in \mathbb{D}$ with $\left|S_{g}\left(w_{0}\right)\right|^{2}\left(1-\left|w_{0}\right|^{2}\right)>\delta$. Thus, we can find a specific positive constant $k=k(\delta)<1$, for which

$$
\left|S_{g}(w)\right|^{2}\left(1-|w|^{2}\right)>\frac{\delta}{32},
$$

whenever $w \in \mathbb{D}\left(w_{0}, k\left(1-\left|w_{0}\right|^{2}\right)\right)$.

Next, some estimations concerning $\sum_{m} \ell[K(B)]$ will be given. For this result, suppose that

$$
T\left(\varphi_{a}, \omega\right)=\frac{K\left(1-\left|\varphi_{a}(w)\right|^{2}\right)}{\omega(1-|w|)} .
$$

Lemma 7. Assume that $p, \varepsilon, \delta$ are positive constants. Hence, for $\lambda_{1}, \lambda_{2}>0$, we have that

$$
\sum_{m} \ell\left[K\left(B Q_{m}\right)\right] \leq \lambda_{1}+\lambda_{2} \int_{\mathbb{D}}\left|S_{g}(w)\right|^{p}\left(1-|w|^{2}\right)^{2 p-2} T\left(\varphi_{a}, \omega\right) d \sigma_{w} .
$$

Proof. Suppose that $B$ is a maximal-type square such that $\ell(B) \neq 1 / 2$. Thus, $\tilde{B}$ is a maximal-type bad square; hence, we can find $w_{0} \in T(\tilde{B})$ with

$$
\left(1-|w|^{2}\right)^{2}\left|S_{g}\left(w_{0}\right)\right|>\delta .
$$

Therefore, using Lemma 6 , we can find a disc $\mathbb{D}_{w_{0}}=$ $\mathbb{D}\left(w_{0}, c\left(1-\left|w_{0}\right|^{2}\right)\right) \subset T(\tilde{B})$ such that

$$
\left(1-|w|^{2}\right)^{2}\left|S_{g}(w)\right|>\frac{\delta}{32}, \forall w \in \mathbb{D}_{w_{0}} .
$$

Therefore, we infer that

$$
\begin{aligned}
\ell[K(B)] \approx \ell[K(\tilde{B})] & \leq \int_{\mathbb{D}_{w_{0}}}\left(1-|w|^{2}\right)^{-2} T\left(\varphi_{a}, \omega\right) d \sigma_{w} \\
& \leq C \int_{\mathbb{D}_{w_{0}}}\left|S_{g}(w)\right|^{p}\left(1-|w|^{2}\right)^{p-2} T\left(\varphi_{a}, \omega\right) d \sigma_{w} .
\end{aligned}
$$

Because the half $T\left(\tilde{B}_{m}\right)$ may be represented by two times only, then there are two specific square types only, $B^{\prime}$ such that $\ell\left(B^{\prime}\right)=1 / 2$; thus (17) is verified. Then, the concerned lemma is therefore established.

\section{Carleson Measure of $K, \omega$-Type and $Q_{K, \omega}(p, q)$ Spaces}

Certain equivalent concerned general conditions for the $n$th derivatives of analytic $Q_{K, \omega}(p, q)$ classes are obtained in the next generalized result.

Theorem 8. Let $0<p<\infty$ and $-2<q<\infty$. Assume that $n$ $\in \mathbb{N}$ and $f \in \mathscr{H}(\mathbb{D})$. Hence, the next general concerned quantities are equivalent:

(1) $f \in Q_{K, \omega}(p, q)$

(2) $\left|f^{(n)}(w)\right|^{p}\left(1-|w|^{2}\right)^{n p-p+q} d \sigma_{w}$ is a Carleson measure of $K, \omega$-type

(3) $\sup _{a \in \mathbb{D}} \int_{\mathbb{D}}\left|f^{(n)}(w)\right|^{p}\left(1-|w|^{2}\right)^{n p-p+q} T\left(K(g(w, a), \omega) d \sigma_{w}\right.$ $<\infty$

(4) $\sup _{a \in \mathbb{D}} \int_{\mathbb{D}}\left|f^{(n)}(w)\right|^{p}\left(1-|w|^{2}\right)^{n p-p+q} T\left(\varphi_{a}, \omega\right) d \sigma_{w}<\infty$.

Proof. (1) $\Longleftrightarrow$ (2). This can be obtained as a corresponding concerned part in Theorem 2 (see [13]).

$(1) \Longleftrightarrow(3)$. This can be deduced as the proof of Theorem 1 in [13] by very simple and minor specific modifications

$(2) \Longleftrightarrow(4)$. Using Lemma 5 for $d \mu(w)=\left|f^{(n)}(w)\right|^{p}$ $\left(1-|w|^{2}\right)^{n p-p+q} d \sigma_{w}$, then $\mu$ is a concerned Carleson measure of $K, \omega$-type if and only if

$$
\begin{aligned}
\sup _{a \in \mathbb{D}} \int_{\mathbb{D}} T\left(\varphi_{a}, \omega\right) d \mu(w)= & \sup _{a \in \mathbb{D}} \int_{\mathbb{D}}\left|f^{(n)}(w)\right|^{p}\left(1-|w|^{2}\right)^{n p-p+q} T \\
& \cdot\left(\varphi_{a}, \omega\right) d \sigma_{w}<\infty .
\end{aligned}
$$

Thus, the concerned proof is completely finished. 
For the compact Carleson measure of $K$, $\omega$-type, the following interesting result can be obtained.

Theorem 9. Let $p \in(0, \infty)$ and $q \in(-2, \infty)$. Suppose also $n$ $\in \mathbb{N}$ and the concerned analytic function $f \in \mathscr{H}(\mathbb{D})$. Then, the next essential specific statements are equivalent:

(a) $f \in Q_{K, \omega, 0}(p, q)$

(b) $\left|f^{(n)}(w)\right|^{p}\left(1-|w|^{2}\right)^{n p-p+q} d \sigma_{w}$ is a concerned compact Carleson measure of $K$, $\omega$-type

(c) $\lim _{|a| \rightarrow 1} \int_{\mathbb{D}}\left|f^{(n)}(w)\right|^{p}\left(1-|w|^{2}\right)^{n p-p+q} T\left(K(g(w, \omega)) d \sigma_{w}\right.$ $=0$

(d) $\left.\lim _{|a| \rightarrow 1} \int_{\mathbb{D}}\left|f^{(n)}(w)\right|^{p}\left(1-|w|^{2}\right)^{n p-p+q} T\left(\varphi_{a}, \omega\right)\right) d \sigma_{w}=0$.

Now, we give the following interesting lemma which connects Carleson measure of $K, \omega$-type, Schwarzian derivative and the pre-Schwarzian derivative.

Lemma 10. Let $1 \leq p<\infty$, and suppose that $q \in(-2, \infty)$ with $q-p \leq-2$ and assume that $f=\ln g^{\prime} \in \mathscr{B}_{0}$. Then, if

$$
\left|S_{g}(w)\right|^{p}\left(1-|w|^{2}\right)^{p+q} d \sigma_{w}
$$

is a concerned $K, \omega$-Carleson measure, we have that $\left|N_{g}(w)\right|^{p}\left(1-|w|^{2}\right)^{q} d \sigma_{w}$ is a concerned $K, \omega$-Carleson measure too.

Proof. Since $S_{g}(w)=N_{g}^{\prime}(w)-1 / 2\left(N_{g}^{\prime}(w)\right)^{2}$, then by Theorem 8 , we deduce

$$
\left|N_{g}^{\prime}(w)\right|^{p}\left(1-|w|^{2}\right)^{q} d \sigma_{w}
$$

is a concerned Carleson measure of $K, \omega$-type $\Longleftrightarrow$

$$
\left|N_{g}^{\prime}(w)\right|^{p}\left(1-|w|^{2}\right)^{p+q} d \sigma_{w}
$$

is a Carleson measure of $K, \omega$-type, and that $\left(1-|w|^{2}\right) \mid N_{g}$ $(w) \mid \leq e^{2}$ for every $w \in \mathbb{D}$. Thus, for any $1 \leq p<\infty$, we obtain that

$$
\begin{aligned}
I(a)= & \int_{\mathbb{D}}\left|N_{g}^{\prime}(w)\right|^{p}\left(1-|w|^{2}\right)^{p+q} T\left(\varphi_{a}, \omega\right) d \sigma_{w} \\
\leq & 2^{p-1} \int_{\mathbb{D}}\left|S_{g}(w)\right|^{p}\left(1-|w|^{2}\right)^{p+q} T\left(\varphi_{a}, \omega\right) d \sigma_{w} \\
& +\frac{1}{2} \int_{\mathbb{D}}\left|N_{g}^{\prime}(w)\right|^{2 p}\left(1-|w|^{2}\right)^{p+q} T\left(\varphi_{a}, \omega\right) d \sigma_{w} .
\end{aligned}
$$

Suppose that $N_{g}$ is continuous function on the closed unit disc $\overline{\mathbb{D}}$; because if the claim is not verified, we will lose the dilatations of $\left(N_{g}\right)_{r}(z)=N_{g}(r w)$; then, the proof can be ended by letting $r \longrightarrow 1$.

Because $h=\ln g^{\prime} \in \mathscr{B}_{0}$, for any $\varepsilon>0$, there exists $r_{\varepsilon}$ for which $|w|>r_{\varepsilon}$; we thus obtain that $\left|N_{g}(w)\right|\left(1-|w|^{2}\right)<\varepsilon$, with

$$
\begin{aligned}
\int_{\mathbb{D}} \mid & \left.N_{g}(w)\right|^{2 p}\left(1-|w|^{2}\right)^{p+q} T\left(\varphi_{a}, \omega\right) d \sigma_{w} \\
= & \int_{|w|>r_{\varepsilon}}\left|N_{g}(w)\right|^{2 p}\left(1-|w|^{2}\right)^{p+q} T\left(\varphi_{a}, \omega\right) d \sigma_{w} \\
& \quad+\int_{|w| \leq r_{\varepsilon}}\left|N_{g}(z)\right|^{2 p}\left(1-|w|^{2}\right)^{p+q} T\left(\varphi_{a}, \omega\right) d \sigma_{w} \\
= & J_{1}(a)+J_{2}(a) .
\end{aligned}
$$

Thus, for some $k=k(p, q)$, we infer that

$$
\begin{aligned}
J_{1}(a) & =\int_{|w|>r_{\varepsilon}}\left|N_{g}^{\prime}(z)\right|^{2 p}\left(1-|w|^{2}\right)^{p+q} T\left(\varphi_{a}, \omega\right) d \sigma_{w} \\
& \leq \varepsilon^{p} \int_{\mathbb{D}}\left|N_{g}^{\prime}(z)\right|^{p}\left(1-|w|^{2}\right)^{q} T\left(\varphi_{a}, \omega\right) d \sigma_{w} \\
& \leq C \varepsilon^{p} \int_{\mathbb{D}}\left|N_{g}^{\prime}(z)\right|^{p}\left(1-|w|^{2}\right)^{p+q} T\left(\varphi_{a}, \omega\right) d \sigma_{w} \\
& =C \varepsilon^{p} J(a) .
\end{aligned}
$$

Because $q \leq p-2$, for every $a \in \mathbb{D}$, we then obtain that

$$
\begin{aligned}
J_{2}(a) & =\int_{|w| \leq r_{\varepsilon}}\left|N_{g}(z)\right|^{2 p}\left(1-|w|^{2}\right)^{p+q} T\left(\varphi_{a}, \omega\right) d \sigma_{w} \\
& \leq e^{4 p} \int_{|w| \leq r_{\varepsilon}}\left(1-|w|^{2}\right)^{q-p} T\left(\varphi_{a}, \omega\right) d \sigma_{w} \leq \frac{e^{2 p}}{\left(1-r_{\varepsilon}{ }^{2}\right)^{p-q}} .
\end{aligned}
$$

letting $\varepsilon$ be so small such that $1-C \varepsilon^{p} / 2>0$. Then,

$$
\begin{aligned}
\left(1-\frac{C \varepsilon^{p}}{2}\right) J(a) \leq & 2^{p-1} \int_{\mathbb{D}}\left|S_{g}(w)\right|^{p}\left(1-|w|^{2}\right)^{p+q} T\left(\varphi_{a}, \omega\right) d \sigma_{w} \\
& +\frac{e^{4 p}}{2\left(1-r_{\varepsilon}^{2}\right)^{p-q}},
\end{aligned}
$$

Since $\left|S_{g}(w)\right|^{p}\left(1-|w|^{2}\right)^{p+q} d \sigma_{w}$ is a concerned Carleson measure of $K, \omega$-type, after considering the supremum over $a \in \mathbb{D}$ of (29), we deduce that

$$
\sup _{a \in \mathbb{D}} J(a)=\sup _{a \in \mathbb{D}} \int_{\mathbb{D}}\left|N_{g}^{\prime}(w)\right|^{p}\left(1-|w|^{2}\right)^{p+q} T\left(\varphi_{a}, \omega\right) d \sigma_{w}<\infty .
$$

Using Theorem 8, we infer that $\left|N_{g}(w)\right|^{p}\left(1-|w|^{2}\right)^{q} d \sigma_{w}$ is a concerned Carleson measure of $K, \omega$-type too. The proof is therefore completely obtained. 
The following essential and interesting proposition shall be given.

Proposition 11. Let $1 \leq p<\infty$ and $-2<q<\infty$. When $f=l$ $n g^{\prime} \in \mathbb{Q}_{K, \omega}(p, q)$, then

$$
\left|S_{g}(w)\right|^{p}\left(1-|w|^{2}\right)^{p+q} d \sigma_{w}
$$

is a Carleson measure of $K, \omega$-type.

Proof. Since

$$
\left\|\ln g^{\prime}\right\|_{\mathscr{B}}=\sup _{a \in \mathbb{D}}\left(1-|w|^{2}\right)\left|N_{g}(w)\right| \leq e^{3} .
$$

In view of Theorem 8 for $n=1$ and $f=\ln g^{\prime}$, we then infer that

$$
f=\operatorname{lng}^{\prime} \in Q_{K, \omega}(p, q) \Longleftrightarrow\left|N_{g}(w)\right|^{p}\left(1-|w|^{2}\right)^{q} d \sigma_{w}
$$

is a Carleson measure of $K, \omega$-type.

Moreover, using Theorem 8 with $n=2$, we deduce that

$$
\left|N_{g}^{\prime}(w)\right|^{p}\left(1-|w|^{2}\right)^{p+q} d \sigma_{w}
$$

is a concerned Carleson measure of $K, \omega$-type.

Therefore, for $p \geq 1$, we have that

$$
\left|S_{g}(w)\right|^{p} \leq 2^{p-1}\left|N_{g}^{\prime}(w)\right|^{p}+\frac{1}{2}\left|N_{g}^{\prime}(w)\right|^{2 p} .
$$

Hence,

$$
\begin{aligned}
\left|S_{g}(w)\right|^{p}\left(1-|w|^{2}\right)^{p+q} \leq & 2^{p-1}\left|N_{g}^{\prime}(w)\right|^{p}\left(1-|w|^{2}\right)^{p+q} \\
& +\frac{1}{2}\left\|\log g^{\prime}\right\|_{\mathscr{B}}\left|N_{g}(w)\right|^{p}\left(1-|w|^{2}\right)^{q} .
\end{aligned}
$$

Using (33) and (34), we have

$$
\left|S_{g}(w)\right|^{p}\left(1-|w|^{2}\right)^{p+q} d \sigma_{w}
$$

is a concerned Carleson measure of $K, \omega$-type.

The proof is therefore completely finished.

\section{Results by Logarithmic Characterizations}

We give some logarithmic characterizations for univalent functions belonging to the classes

$$
S_{W}=\left\{\ln g^{\prime}: g \in \mathscr{U}, \quad \ln g^{\prime} \in W\right\},
$$

where $W$ is the weighted holomorphic $Q_{K, \omega}(p, q)$ class or the weighted holomorphic $Q_{K, \omega, 0}(p, q)$ class.
Theorem 12. Let $p \in[1, \infty)$ and $q \in(-2, \infty)$ also suppose that $q+2<p$ or $q=p-2$ and $K(t) \equiv 1$. Thus, the following fundamental concerned statements are equivalent:

(I) $\operatorname{lng}^{\prime} \in Q_{K, \omega}(p, q)$

(II) $\operatorname{lng}^{\prime} \in \mathscr{B}_{\omega, 0}$ and $\left|S_{g}(w)\right|^{p}\left(1-|w|^{2}\right)^{p+q} d \sigma_{w}$ is a concerned Carleson measure of $K, \omega$-type.

Proof. Assume that the extended choice of the parameters $p, q$ $\ln g^{\prime} \in Q_{K, \omega}(p, q) \subset \mathscr{B}_{\omega}^{q+2 / p}$. Then, if $q+2<p, Q_{K, \omega}(p, q) \subset$ $\mathscr{B}_{\omega}^{\alpha}$, with $0<\alpha<1$. The concerned proof of (I) be deduced by Lemma 10 as well as Proposition 11 .

Remark 13. When $q=p-2$ and $K(t) \equiv 1, \omega(t) \equiv 1$, that is, the case of the weighted holomorphic Besov-type spaces $B_{p}, 1$ $<p<\infty$, it can be proved clearly by remarking that each of these classes is also inclusive in the little Bloch class $\mathscr{B}_{0}$. This case was presented in [3] too.

Theorem 14. Let $p \in[1, \infty)$ and $q \in(-2, \infty)$ and suppose that $q+2=p$. Thus,

$$
\ln g^{\prime} \in Q_{K, \omega}(p, q) \Longleftrightarrow\left|S_{g}(w)\right|^{p}\left(1-|w|^{2}\right)^{p+q} d \sigma_{w}
$$

is a concerned Carleson measure of $K, \omega$-type.

Proof. Suppose that $\ln g^{\prime} \in Q_{K, \omega}(p, q)$; then, by the help of Proposition 11, we deduce that

$$
\left|S_{g}(w)\right|^{p}\left(1-|w|^{2}\right)^{p+q} d \sigma_{w}
$$

is a concerned Carleson measure of $K, \omega$-type.

Because the equality $q=p-2$, we then obtain that $Q_{K, \omega}(p, q)=Q_{K, \omega}(p, p-2)$. Now, the aim is to prove that when

$$
\left|S_{g}(w)\right|^{p}\left(1-|w|^{2}\right)^{2 p-2} d \sigma_{w}
$$

is a concerned Carleson measure of $K$, $\omega$-type, then $\ln g^{\prime}$ $\in Q_{K, \omega}(p, p-2)$. Thus, we need to prove that

$$
\int_{\mathbb{D}}\left|S_{g}(w)\right|^{p}\left(1-|w|^{2}\right)^{2 p-2} T\left(\varphi_{a}, \omega\right) d \sigma_{w}<\infty
$$

implies that

$$
\int_{\mathbb{D}}\left|N_{g}(w)\right|^{p}\left(1-|w|^{2}\right)^{p-2} T\left(\varphi_{a}, \omega\right) d \sigma_{w}<\infty .
$$

This is equivalent to

$$
\int_{\mathbb{D}}\left|N_{g}^{\prime}(w)\right|^{p}\left(1-|w|^{2}\right)^{2 p-2} T\left(\varphi_{a}, \omega\right) d \sigma_{w}<\infty
$$


Since $\left|N_{g}^{\prime}(w)\right|^{p} \leq 2^{p-1}\left|S_{g}(w)\right|^{p}+1 / 2\left|N_{g}(z)\right|^{2 p}$, then we obtain that

$$
\begin{aligned}
\int_{\mathbb{D}}\left|N_{g}^{\prime}(w)\right|^{p}\left(1-|w|^{2}\right)^{2 p-2} T\left(\varphi_{a}, \omega\right) d \sigma_{w} \\
\leq 2^{p-1} \int_{\mathbb{D}}\left|S_{g}(w)\right|^{p}\left(1-|w|^{2}\right)^{2 p-2} T\left(\varphi_{a}, \omega\right) d \sigma_{w} \\
\quad+\frac{1}{2} \int_{\mathbb{D}}\left|N_{g}(w)\right|^{2 p}\left(1-|w|^{2}\right)^{2 p-2} T\left(\varphi_{a}, \omega\right) d \sigma_{w} .
\end{aligned}
$$

Let us now suppose that $N_{g}$ is a specific concerned continuous function on $\overline{\mathbb{D}}$, which hence occupy that $r \longrightarrow 1$ for the end of the concerned proof.

Calculating the following specific integral

$$
I_{N_{g}^{2}}(\mathbb{D})=\int_{\mathbb{D}}\left|N_{g}(w)\right|^{2 p}\left(1-|w|^{2}\right)^{2 p-2} T\left(\varphi_{a}, \omega\right) d \sigma_{w}
$$

For $\varepsilon, \delta>0$, suppose that

$$
\begin{aligned}
D & =\left\{w \in \mathbb{D}:\left|N_{g}(w)\right|\left(1-|w|^{2}\right)<\varepsilon\right\}, \\
D_{1} & =\left\{w \in \mathbb{D}:\left|S_{g}(w)\right|\left(1-|\mathrm{w}|^{2}\right)^{2}>\delta\right\}, \\
\Delta=\mathbb{D} \backslash\left(D \cup D_{1}\right) & =\left\{w \in \mathbb{D}:\left|N_{g}(w)\right|\left(1-|w|^{2}\right) \geq \varepsilon,\left|S_{g}(w)\right|\left(1-|w|^{2}\right)^{2} \leq \delta\right\} .
\end{aligned}
$$

Using Theorem 8, we can find a specific constant $\lambda>0$, for which

$$
\begin{aligned}
& \int_{\mathbb{D}}\left|N_{g}^{\prime}(w)\right|^{p}\left(1-|w|^{2}\right)^{p-2} T\left(\varphi_{a}, \omega\right) d \sigma_{w} \\
& \quad \leq \lambda \int_{\mathbb{D}}\left|N_{g}^{\prime}(w)\right|^{p}\left(1-|w|^{2}\right)^{2 p-2} T\left(\varphi_{a}, \omega\right) d \sigma_{w}
\end{aligned}
$$

Then,

$$
\begin{aligned}
I_{N_{g}^{2}}(D) & =\int_{D}\left|N_{g}^{\prime}(w)\right|^{2 p}\left(1-|w|^{2}\right)^{2 p-2} T\left(\varphi_{a}, \omega\right) d \sigma_{w} \\
& <\varepsilon^{p} \int_{D}\left|N_{g}^{\prime}(w)\right|^{p}\left(1-|w|^{2}\right)^{p-2} T\left(\varphi_{a}, \omega\right) d \sigma_{w} \\
& \leq \varepsilon^{p} \int_{\mathbb{D}}\left|N_{g}^{\prime}(w)\right|^{p}\left(1-|w|^{2}\right)^{p-2} T\left(\varphi_{a}, \omega\right) d \sigma_{w} \\
& \leq \lambda \varepsilon^{p} \int_{\mathbb{D}}\left|N_{g}^{\prime}(w)\right|^{p}\left(1-|w|^{2}\right)^{2 p-2} T\left(\varphi_{a}, \omega\right) d \sigma_{w} .
\end{aligned}
$$

Since $\left|N_{g}(w)\right|\left(1-|w|^{2}\right)<6<e^{2}$, we infer that

$$
\begin{aligned}
I_{N_{g}^{2}}\left(D_{1}\right) & =\int_{D_{1}}\left|N_{g}(w)\right|^{2 p}\left(1-|w|^{2}\right)^{2 p-2} T\left(\varphi_{a}, \omega\right) d \sigma_{w} \\
& <e^{4 p} \int_{D_{1}}\left(1-|w|^{2}\right)^{-2} T\left(\varphi_{a}, \omega\right) d \sigma_{w}
\end{aligned}
$$

$$
\begin{aligned}
& <\frac{e^{4 p}}{\delta^{p}} \int_{D_{1}}\left|S_{g}(w)\right|^{p}\left(1-|w|^{2}\right)^{2 p-2} T\left(\varphi_{a}, \omega\right) d \sigma_{w} \\
& \leq \frac{e^{4 p}}{\delta^{p}} \int_{\mathbb{D}}\left|S_{g}(z)\right|^{p}\left(1-|w|^{2}\right)^{2 p-2} T\left(\varphi_{a}, \omega\right) d \sigma_{w} .
\end{aligned}
$$

Then,

$$
I_{N_{g}^{2}}(\Delta)=\int_{\Delta}\left|N_{g}(w)\right|^{2 p}\left(1-|w|^{2}\right)^{2 p-2} T\left(\varphi_{a}, \omega\right) d \sigma_{w}
$$

Let us consider the sequence $\left\{B_{m}\right\}$ of Carleson bad boxes, then

$$
\begin{aligned}
I_{N_{g}^{2}}(\Delta) & =\int_{\Delta}\left|N_{g}(z)\right|^{2 p}\left(1-|w|^{2}\right)^{2 p-2} T\left(\varphi_{a}, \omega\right) d \sigma_{w} \\
& <e^{4 p} \int_{\Delta} T\left(\varphi_{a}, \omega\right) \frac{d \sigma_{w}}{\left(1-|w|^{2}\right)^{2}} \\
& \leq e^{4 p} \sum_{m} \int_{T\left(B_{m}\right)} T\left(\varphi_{a}, \omega\right) \frac{d \sigma_{w}}{\left(1-|w|^{2}\right)^{2}} \\
& \leq e^{4 p} C \sum_{m} \ell\left[K\left(B_{m}\right)\right] .
\end{aligned}
$$

For $\varepsilon>0$ such that $\lambda \varepsilon^{p}<1$, we infer that

$$
\begin{aligned}
\int_{\mathbb{D}} \mid & \left.N_{g}^{\prime}(w)\right|^{p}\left(1-|w|^{2}\right)^{2 p-2} T\left(\varphi_{a}, \omega\right) d \sigma_{w} \\
\leq & 2^{p-1} \int_{\mathbb{D}}\left|S_{g}(z)\right|^{p}\left(1-|w|^{2}\right)^{2 p-2} T\left(\varphi_{a}, \omega\right) d \sigma_{w} \\
& +\frac{\lambda \varepsilon^{p}}{2} \int_{\mathbb{D}}\left|N_{g}^{\prime}(z)\right|^{p}\left(1-|w|^{2}\right)^{2 p-2} T\left(\varphi_{a}, \omega\right) d \sigma_{w} \\
& +\frac{e^{4 p}}{2 \delta^{p}} \int_{\mathbb{D}}\left|S_{g}(z)\right|^{p}\left(1-|w|^{2}\right)^{2 p-2} T\left(\varphi_{a}, \omega\right) d \sigma_{w} \\
& +e^{4 p} C \sum_{m} \ell\left[K\left(B_{m}\right)\right] .
\end{aligned}
$$

By Lemma 7, we deduce that

$$
\sum_{m} \ell\left[K\left(B_{m}\right)\right] \leq C_{1}+C_{2} \int_{\mathbb{D}}|S \varphi(w)|^{p}\left(1-|w|^{2}\right)^{p-2} T\left(\varphi_{a}, \omega\right) d \sigma_{w}
$$

Letting $C$ appear as a global concerned positive constant, we may infer that

$$
\begin{aligned}
& \left(1-\frac{\lambda \varepsilon^{p}}{2}\right) \int_{\mathbb{D}}\left|N_{g}^{\prime}(w)\right|^{p}\left(1-|w|^{2}\right)^{2 p-2} T\left(\varphi_{a}, \omega\right) d \sigma_{w} \\
& \leq C \int_{\mathbb{D}}\left|S_{g}(w)\right|^{p}\left(1-|w|^{2}\right)^{2 p-2} T\left(\varphi_{a}, \omega\right) d \sigma_{w} .
\end{aligned}
$$


Therefore,

$$
\int_{\mathbb{D}}\left|S_{g}(w)\right|^{p}\left(1-|w|^{2}\right)^{2 p-2} T\left(\varphi_{a}, \omega\right) d \sigma_{w}<\infty,
$$

which gives that

$$
\int_{\mathbb{D}}\left|N_{g}^{\prime}(w)\right|^{p}\left(1-|w|^{2}\right)^{2 p-2} T\left(\varphi_{a}, \omega\right) d \sigma_{w}<\infty
$$

This is equivalent to $\ln g^{\prime} \in Q_{K, \omega}(p, q)$, and this finishes the proof.

\section{Conclusions}

Revealing studies on the theory of weighted function spaces evolving the operator theory which includes important and useful studies could enhance research on both theories. In this concerned manuscript, the investigations of emission evolving a class of operators on a general space of weighted functions are then studied. Typically, the possibility of acting the Schwarzian derivative evolving certain Q-type classes is explored. We have revealed that the majority of proof methods supply a snapshot of the role of the Schwarzian derivatives under properties of the weighted class of function spaces. As the definition of the weighted holomorphic Q-type of function class structure, some changes in weights and in the derivative part, the amount of required results entirely is large, and it leads to an efficient clear utility.

To this end, we propose interesting characterizations, a novel of Schwarzian differentially private evolving Q-type of function class releasing the derivative algorithm which reduces the scales and tool up a clear utility.

\section{Data Availability}

We have not apply or consider any data during this concerned current research.

\section{Conflicts of Interest}

The authors of this concerned study declare completely that they have no any competing of interests.

\section{Acknowledgments}

The first author thanks the Taif University researchers for the support (Project number TURSP-2020/159), Taif University, Saudi Arabia. S. Omran thanks the Academy of Scientific Research and Technology (ASRT), Egypt, for supporting him with the project(6407).

\section{References}

[1] F. Pérez-González and J. Rättyä, "Dirichlet and VMOA domains via Schwarzian derivative," Journal of Mathematical Analysis and Applications, vol. 359, no. 2, pp. 543-546, 2009.

[2] C. Pommerenke, Boundary Behaviour of Conformal Maps, Springer-Verlag, 1992.
[3] C. Pommerenke, Univalent Functions, Vandenhoeck and Ruprecht, Göttingen, 1975.

[4] A. El-Sayed Ahmed and M. Youssif, "Classes of weighted tent function spaces and mixed norms with some applications," Italian Journal of Pure and Applied Mathematics, vol. 43, pp. 402-415, 2020.

[5] R. A. Rashwan, A. El-Sayed Ahmed, and A. Kamal, "Some characterizations of weighted Bloch space," European Journal of Pure and Applied Mathematics, vol. 2, pp. 250-267, 2009.

[6] R. A. Rashwan, A. E.-S. Ahmed, and A. Kamal, "Integral characterizations of weighted Bloch spaces and $Q_{K, \omega}(p, q)$ spaces," Mathematica Cluj, vol. 51, no. 1, pp. 63-76, 2009.

[7] A. E.-S. Ahmed and A. Kamal, "Carleson measure characterization on analytic $Q_{K}(p, q)$ spaces," Journal of international mathematical virtual institute, vol. 3, pp. 1-21, 2013.

[8] A. E.-S. Ahmed and A. Kamal, "Generalized composition operators on $Q_{K, \omega}(p, q)$ spaces," Mathematical Sciences, vol. 6, no. 1, p. 14, 2012.

[9] H. Li and T. Ma, "Generalized composition operators from $B_{\mu}$ spaces to $Q_{K, \omega}(p, q)$ spaces," Abstract and Applied Analysis, vol. 2914, Article ID 897389, 2014.

[10] J. Long and D. Shang, "Composition operators from Zygmund spaces into $Q_{K, \omega}(p, q)$ spaces," Mathematica Aeterna, vol. 3, no. 8, pp. 679-692, 2013.

[11] X. Pei and H. Wulan, "Distance of Bloch-type function to $Q_{K}(p, q)$ space," Complex Variables and Elliptic Equations, vol. 64 , no. 9, pp. 1568-1581, 2019.

[12] H. Wulan and J. Zhou, " $Q_{K}$ type spaces of analytic functions," Journal of Function Spaces, vol. 4, 84 pages, 2006.

[13] H. Wulan and J. Zhou, "The higher order derivatives of $Q_{K}$ type spaces," Journal of Mathematical Analysis and Applications, vol. 332, no. 2, pp. 1216-1228, 2007.

[14] A. E.-S. Ahmed, "Lacunary series in quaternion $\mathrm{B}^{\mathrm{p}, \mathrm{q}}$ spaces," Complex Variables and Elliptic Equation, vol. 54, no. 7, pp. 705-723, 2009.

[15] A. El-Sayed Ahmed, "Hyperholomorphic Q-classes," Mathematical and Computer Modelling, vol. 55, no. 3-4, pp. 14281435, 2012.

[16] A. El-Sayed Ahmed, S. Omran, and S. Omran, "Weighted classes of quaternion-valued functions," Banach Journal of Mathematical Analysis, vol. 6, no. 2, pp. 180-191, 2012.

[17] A. El-Sayed Ahmed and S. Omran, "Extreme points and some quaternion valued functions in the unit ball of $\mathbb{R}^{3}$," Advances in Applied Clifford Algebras, vol. 28, no. 1, 2018.

[18] A. El-Sayed Ahmed and M. A. Bakhit, "Characterizations involving Schwarzian derivative in some analytic function spaces," Mathematical Sciences, vol. 7, no. 1, p. 43, 2013.

[19] C. J. Bishop and P. W. Jones, "Harmonic measure, L ${ }^{2}$ estimates and the Schwarzian derivative," Journal D' analyse Mathematique, vol. 62, no. 1, pp. 77-113, 1994.

[20] V. Bolotnikov, "Several inequalities for the Schwarzian derivative of bounded analytic functions," Complex Variables and Elliptic Equations, vol. 64, no. 7, pp. 1093-1102, 2018.

[21] M. Chuaqui and B. Osgood, "Sharp distortion theorems associated with the Schwarzian derivative," Journal of the London Mathematical Society, vol. 48, pp. 289-298, 1993.

[22] J. Pau and J. Á. Peláez, "Logarithms of the derivative of univalent functions in Qp spaces," Journal of Mathematical Analysis and Applications, vol. 350, no. 1, pp. 184-194, 2009. 
[23] N. Zorboska, "Schwarzian derivative and general Besov-type domains," Journal of Mathematical Analysis and Applications, vol. 379, no. 1, pp. 48-57, 2011.

[24] R. Aulaskari, D. A. Stegenga, and J. Xiao, "Some subclasses of BMOA and their characterization in terms of Carleson measures," The Rocky Mountain Journal of Mathematics, vol. 26, no. 2, pp. 485-506, 1996.

[25] M. Essén, H. Wulan, and J. Xiao, "Several function-theoretic aspects of Möbius invariant $Q_{K}$ spaces," Journal of Functional Analysis, vol. 230, pp. 78-115, 2006. 